\title{
A quality indicator set for use in rehabilitation team care of people with rheumatic and musculoskeletal diseases; development and pilot testing
}

\author{
Inger Johansen ${ }^{1,2^{*}}$ (D) Mari Klokkerud ${ }^{1}$, Audny Anke ${ }^{3,4}$, Janne-Birgitte Børke ${ }^{10}$, Thomas Glott ${ }^{9}$, Uno Hauglie ${ }^{11}$, \\ Audhild Høyem ${ }^{13}$, Atle Klovning ${ }^{2}$, Karin Anna Lande ${ }^{12}$, Mona Larsen ${ }^{8}$, Jan Egil Nordvik ${ }^{9}$, Sigrid H. Wigers ${ }^{7}$, \\ Irene Øyeflaten ${ }^{5,6}$, Kaare Birger Hagen ${ }^{1}$ and Ingvild Kjeken ${ }^{1}$
}

\begin{abstract}
Background: Systems for monitoring effectiveness and quality of rehabilitation services across health care levels are needed. The purpose of this study was to develop and pilot test a quality indicator set for rehabilitation of rheumatic and musculoskeletal diseases.

Methods: The set was developed according to the Rand/UCLA Appropriateness Method, which integrates evidence review, in-person multidisciplinary expert panel meetings and repeated anonymous ratings for consensus building. The quality indicators were pilot-tested for overall face validity and feasibility in 15 specialist and 14 primary care rehabilitation units. Pass rates (percentages of "yes") of the indicators were recorded in telephone interviews with 29 unit managers (structure indicators), and 164 patients (process and outcome indicators). Time use and participants' numeric rating of face validity $(0-10,10=$ high validity) were recorded.

Results: Nineteen structure, 12 process and five outcome indicators were developed and piloted. Mean (range) sum pass rates for the structure, process and outcome indicators were 59\%(84\%), 66\%(100\%) and 84\%(100\%), respectively. Mean (range) face validity score for managers/patients was 8.3 (8)/7.9 (9), and mean answering time was 6.0/5.5 min. The final indicator set consists of 19 structure, 11 process and three outcome indicators.

Conclusion: To our knowledge this is the first quality indicator set developed for rehabilitation of rheumatic and musculoskeletal diseases. Good overall face validity and a feasible format indicate a set suitable for monitoring quality in rehabilitation. The variation in pass rates between centers indicates a potential for quality improvement in rheumatic and musculoskeletal rehabilitation in Norway.
\end{abstract}

Keywords: Rehabilitation, Musculoskeletal diseases, Quality indicators, Health care, Delphi technique

\section{Background}

Rheumatic and musculoskeletal diseases (RMDs) are among the most regular disorders in the general population and have a large economic and social impact on societies [1]. Due to increased life expectancy and overweight,

\footnotetext{
* Correspondence: inger.johansen@medisin.uio.no

${ }^{1}$ National Advisory Unit on Rehabilitation in Rheumatology, Diakonhjemmet Hospital, P.O. Box 23, 0319 Oslo, Norway

${ }^{2}$ Department of General Practice, Institute of Health and Society, Faculty of Medicine, University of Oslo, P.O. Box 1130 Blindern, 0318 Oslo, Norway Full list of author information is available at the end of the article
}

the number of people with RMDs will rise in the coming decades [2]. Even though biological medicines have revolutionized the treatment of inflammatory rheumatic diseases in the past one-and-a-half decades, pharmacological treatment of the majority of RMDs still mainly targets alleviation of symptoms [3, 4]. Successful management of these diseases therefore is strongly dependent on the patient's ability to implement life-style changes and self-management strategies, often introduced through rehabilitation programmes. Several studies have shown that patients with RMDs benefit from rehabilitation, but the

(c) The Author(s). 2019 Open Access This article is distributed under the terms of the Creative Commons Attribution 4.0 International License (http://creativecommons.org/licenses/by/4.0/), which permits unrestricted use, distribution, and 
effect seems to decline over time, and most people are back to their initial health status six to twelve months after discharge [5-7].

When it comes to the organization of rehabilitation in Norway, healthcare statutes push for a shift in the direction of shorter in-patient stays or day programmes in specialized care, and more rehabilitation and better follow-up in primary care. However, several recent reports conclude that the quality of rehabilitation services in general is low, characterized by a lack of coordination across levels of care, and with divergences in content and quality, especially in primary care. Furthermore, the same reports underline that systems for monitoring effectiveness and quality across health care levels are needed $[8,9]$. One method to monitor the quality of patient care and to clarify areas of improvement is to develop quality indicators (QIs) of care [10]. QIs can be defined as "measurable elements of practice performance for which there is evidence or consensus that it can be used to assess the quality, and hence to change the quality, of care provided" [11]. The purpose of healthcare quality indicators is to serve as i) a basis for management for authorities and owners, ii) tools for internal quality improvement and iii) support for patients in choosing service providers [10]. QIs are related to structure (the settings in which care occurs), process (what is actually done in giving and receiving care), or outcome of health care [12].

QI sets to manage different rheumatic diseases have been developed [13-18]. Some of the QIs are adapted to a specific diagnosis, others are more generic. However, most quality sets mainly include process indicators, some of them related to rehabilitation and some not, and the development processes were suboptimal [15, 18]. Comprehensive and resource demanding quality assurance and accreditation systems for social and rehabilitation services exist [19-21], as do human rights based indicator framework systems to assess countries' efforts to strengthen rehabilitation provision and policy [22]. Nevertheless, to our knowledge, no valid and feasible indicator set has been developed for use in rehabilitation of RMDs, covering both structure, process and outcome quality and using appropriate methodology.

The purpose of this project was to develop and pilot test a set of QIs for use in rehabilitation team care of people with RMDs.

\section{Methods}

We used a design based on the Rand/UCLA Appropriateness Method, which has been widely used to develop healthcare QIs [23]. The method integrates evidence review, in-person multidisciplinary expert panel meetings and repeated anonymous ratings for consensus building $[23,24]$, and was conducted in four separate steps: i) preparatory phase, ii) consensus building, iii) pilot testing, and iv) consensus of the final QI set.

\section{Preparatory phase Definitions and criteria}

A broad national working group of nine members was established, approved by The Norwegian Health Directorate. The sampling strategy was non-random, aiming for a broad and relevant composition for the project. Both genders (three men, six women) and different geographic areas of Norway were represented. The professions were physician, nurse, physical- and occupational therapist (two of each), representing clinicians, researchers and administrators. The Norwegian Rheumatism Association nominated a patient representative. Four of the working group members later became participants of the broader expert panel.

The working group prepared the consensus process in an initial meeting. Rehabilitation was defined according to the definition provided by the Norwegian Ministry of Health and Social Affairs as "... a process, or set of processes, which is (are) planned and limited in time, where several professions or services cooperate in assisting the individual user in his or her own efforts to achieve best possible functioning and coping capabilities, and promoting independence and participation in society" [25]. Further, rehabilitation was understood as a process or trajectory that reaches across levels of care. The core professions in rehabilitation in primary care rehabilitation were agreed to be physical therapist(s), occupational therapist(s), nurse(s) and physician(s), and in specialist care, at least one more profession in addition to these.

\section{Systematic search for QIs in rehabilitation}

We conducted a systematic literature search in Medline, Embase, Amed, PsycINFO, PubMed and Cochrane from January 1980 to October 2014 for existing QIs in rehabilitation. Search terms were Quality Indicators, Health Care, Structure or Process or Outcome, Quality and Rehabilitation. The search was not limited to diagnostic groups or study design. We included only publications available in English, German, or Scandinavian languages. A total of 660 publications were identified, of which 12 described QIs for rehabilitation. These concerned rehabilitation in general $(n=4)$, rheumatoid arthritis or osteoarthritis $(n=5)$, and low back pain, osteoporosis or older people (1 publication each). We found no publications describing a QI set for rehabilitation of RMDs. However, several main themes for quality measurement were identified. This informed the process of generating a set of proposed quality domains, which later in the process were operationalized into specific QIs. The literature search was updated in March 2019. Eighty-one new publications were identified, of which 
three described QIs or quality measures for different managements of rheumatoid arthritis and osteoarthritis [16], psoriatic arthritis [17] and inflammatory arthritis [18]. One paper described human rights based indicator framework systems to assess countries' efforts to strengthen rehabilitation provision and policy [22].

\section{The expert panel}

A broad national expert panel comprising 18 members was established (Table 1) [23]. To be deemed an expert, she/he had to have profound clinical, research and/or administrative experience and knowledge and be seen as a trusted expert in the field of rehabilitation. The sampling strategy was non-random selection aiming to seek participants from different geographical areas, levels of care, and with different professional training, position and rehabilitation experience. The patient representatives in the panel had rheumatoid arthritis and low-back pain and were nominated by the Norwegian Rheumatism Association and the Norwegian Back Association.

\section{Consensus building}

In an introduction meeting, the expert panel was informed about the aims and frames of the project, results of the literature search and proposed quality domains, and was invited to propose additional domains. Thereafter, a second literature search was performed, related to empirical evidence for the proposed domains. The search terms were different synonyms of the proposed domains: "population selection criteria"; "discharge criteria"; "length of stay"; "bio-psycho-social assessment"; "patient participation"; "patient-centred"; "arena"; "facilities"; "equipment"; "systematic and targeted rehabilitation"; "staffing"; "multidisciplinary competence", and "outcome". All searches were combined with different search terms for rehabilitation; in addition, the search for outcome was combined with the search terms "patient satisfaction", "rehabilitation outcome" or "clinical outcome" or "treatment outcome". The searches were not limited to any diagnostic group.
Publications related to empirical evidence for the proposed domains were included in a reference list along with all relevant reports and recommendations. Subsequently, we prepared one fact sheet for each proposed quality domain including the rationale, definition and categorization of the domain, history and evidence with references to relevant literature, relevant patient populations and health care levels. Based on the reference list, the fact sheets and their own expertise, the panel members, in two voting rounds, reached a consensus on a set of quality domains.

\section{Round 1}

The fact sheets describing each quality domain and the reference list were distributed to all panel members by e-mail. Members anonymously rated each domain according to the following OECD criteria in the Health Care Quality Project [26]: Importance of what is being measured, scientific soundness of the measure (validity), usefulness (three sub-scales: $i$ susceptibility of being influenced by the health care system, $i i$ the degree to which the use of the quality measure results in desired outcomes, iii free from errors), and feasibility. Each criterion was scored on a Likert scale from 1 to $9(9=$ high degree of agreement).

\section{Selection criteria}

For each quality domain, medians of the nine point Likert scores were calculated and the domains were classified into three levels of appropriateness: 1) "appropriate" was defined as a panel median of 7-9 for all criteria. 2) "uncertain" (and included in the next voting round) was defined as a panel median of $\geq 6$ in up to two criteria and a median of $\geq 7$ in the rest, and 3) "inappropriate" (and excluded from further voting rounds) was defined as a panel median lower than the minimum scores for "uncertain".

\section{Round 2}

The expert panel met for a second one-day, in-person meeting. The results from round 1 were discussed,

Table 1 Characteristics of members in the expert panel

\begin{tabular}{|c|c|c|c|c|c|c|c|c|c|c|}
\hline \multirow[t]{2}{*}{ Profession } & \multicolumn{2}{|c|}{ Gender } & \multicolumn{2}{|c|}{ Health care level } & \multicolumn{3}{|c|}{ Professional role ${ }^{a}$} & \multicolumn{3}{|c|}{ Rehabilitation experience } \\
\hline & $\overline{\mathrm{F}^{\mathrm{b}}}$ & $\overline{M^{c}}$ & $\overline{S^{d}}$ & $p^{e}$ & Clinician & Researcher & Admin & RMDs & Vocational & Genera \\
\hline Patient representative & 1 & 1 & & & & & & 2 & & \\
\hline Physical therapist & 4 & 1 & 3 & 2 & 3 & 2 & 2 & 2 & 1 & 2 \\
\hline Occupational therapist & 3 & 1 & 2 & 2 & 2 & 1 & 3 & 1 & & 3 \\
\hline Nurse & 1 & & 1 & 1 & 1 & & 1 & & & 2 \\
\hline Social educator & 1 & & & & & & 1 & & & \\
\hline Physician & 3 & 1 & 4 & & 3 & 3 & 4 & 3 & & 3 \\
\hline Psychologist & & 1 & 1 & & & 1 & 1 & & & 1 \\
\hline
\end{tabular}

${ }^{a}$ Most members had more than one professional role. ${ }^{b}$ Female. ${ }^{c}$ Male. ${ }^{d}$ Specialist care. ${ }^{e}$ Primary care 
and the panel decided to revise the wording and elements of some of the domains. Following this, the domains were assessed a second time, during which the panel members voted on the domains that had been rated "uncertain" in round 1 .

After round 2, the results from the consensus process were presented to the expert group and discussed in a third one-day, in-person meeting. Each appropriate and uncertain quality domain was formulated as one or more specific QIs, and the panel reached consensus on a set of QIs to be pilot tested.

\section{Pilot testing}

The purpose of the pilot testing was to test the face validity (the degree to which the instrument appears to be an adequate reflection of the construct to be measured [27]) and the feasibility of the QI set. Preferably, QIs are to be extracted from existing data sources such as clinical registers or patient records [26]. However, extracting data from the electronic patient records is not yet possible in Norway, and there is only one relevant patient register that covers a limited segment of the RMD population [28]. Therefore, two yes/no questionnaires were developed for the pilot testing; one for rehabilitation unit managers (addressing structure QIs) and one for patients (addressing process and outcome QIs). Several of the structure and process QIs were matched, to be able to align quality assessment from both a system and user perspective. One example of this might be to ask managers if the unit had written procedures in daily use for making an individual rehabilitation plan (structure QI) and to ask the patient if she had received such a plan (process QI).

Rehabilitation units from both specialist and primary care were invited to participate in the pilot testing, using a snowball method whereby expert panellists suggested units to be invited. Both oral and written information about the study was provided. The managers of the rehabilitation units answered the structure QIs in the beginning of the test period, whereas patients answered the process and outcome QIs one to two months after completion of a rehabilitation programme. The questionnaire was first sent by e-mail to the participants, who thereafter gave their answers in a telephone interview conducted by the first author (IJ) or a study secretary.

The time spent answering the questionnaire in the interview was recorded in minutes, and participants' ratings of face validity were recorded on a numeric rating scale $(0-10,10=$ to a large degree $)$ according to the question: To what degree do you think the questions capture important elements in rehabilitation? The participants were also asked to comment on the content and comprehensibility of the questionnaire.
The study was approved by the Data Inspectorate/Data Protection Official (ref.no: 2015/16099). All participants provided written consent.

\section{Reaching consensus on the final QI set}

Results from the pilot test were used as a basis for the discussion in the expert panel in a fourth meeting. Thereafter, the uncertain QIs were rated by the options yes or no for approval/disapproval of each QI in a final anonymous consensus round. The previously uncertain QI was included if $75 \%$ or more of the panel had approved it.

\section{Data analysis}

Data were analysed in the statistical software SPSS version 21. Descriptive data were given in means and medians. QI pass rates (PRs) were calculated in percent. Separate PRs for each QI were calculated by dividing the number of participants passing the QI (reporting "yes") by the total number of participants answering "yes" or "no" for the same QI. Correspondingly, summary PRs were calculated by dividing the total number of QIs passed by each participant by the total number of eligible QIs. Correlations between PRs were analysed by Pearson's $r$ and comparisons of PRs between specialist and primary care by Independent Samples T-test or Mann-Whitney U Test. The statistical significant $p$-value was set at $=<0.05$.

\section{Results}

\section{Consensus building}

\section{Proposed quality domains}

Based on assessment by experts, the initial literature review and relevant reports and recommendations, a first set of 35 quality domains was proposed (Table 2). Of these, 20 were related to structure, 10 to process and five to outcome (Table 2. Figure 1).

The literature searches related to the proposed quality domains identified 7866 publications related to "population selection criteria" ( $n=1306)$, "discharge criteria" and "length of stay" ( $n=900)$, "bio-psycho-social assessment" ( $n=1482)$, "patient participation" and "patient-centred" ( $n=979)$, "arena", "facilities" and "equipment" $(n=823)$, "systematic and targeted rehabilitation" ( $n=782)$, "staffing", "multidisciplinary competence" ( $n=974)$, and "outcome" $(n=620)$. The first author screened the titles and abstracts. A total of 38 articles were deemed relevant according to the quality domains and were included in the reference list, which served as background material for the consensus process.

\section{Voting round 1 and 2}

Fourteen expert panel members (78\%) voted in round 1, and 17 (94\%) in round 2 . The rating rounds resulted in 
Table 2 Main themes of quality and corresponding quality domains. Results of the first two voting rounds

Main themes of quality and corresponding references
Facilities and equipment [15] [25, 29-31]
Rehabilitation professionals; education, training and experience, skills,
dedication and motivation, staffing ratio [15, 25, 29-32]

dedication and motivation, staffing ratio [15, 25, 29-32]

Admission an discharge criteria $[15,25,31,33]$

The intervention; patient education, exercises, assistive devices, medication User involvement in the rehabilitation process [15, 20, 25, 29-31, 34-36]

Health Related Quality of Life

Restored or improved function in $\mathrm{ADL}$

Ability to return to pre-event dwelling

Successful involvement in school, work, leisure and social activities $[25,29$,

A

Arena, universal design

$\frac{\text { Rating }^{\mathrm{e}} \text { Cate-gory }}{\mathrm{S}^{\mathrm{a}}}$

Environment

focus on rehabilitation

S

stimulating, supportive, accessible

$\mathrm{S}$

Equipment

appropriate and sufficient S

of high quality

Minimum number of professions

Access to professions outside ordinary staff S

Adequate staffing $p^{b}$

Admission criteria $\mathrm{S}$

Discharge criteria S

Defined patient target group $\quad * \quad$ S

Use of

validated assessment instruments $\quad * \quad \mathrm{~S}$

instruments covering physical, mental and social * P P

aspects

Multi professional assessment at

admission S

discharge S

Bio-psycho-social assessment at

admission S

discharge S

Initial bio-psycho-social assessment ** P

Procedure for patient participation in

planning and evaluating the $\quad * * \quad S$

rehabilitation process

goal setting $\quad * *$ S

Patient participated in

planning the intervention $\quad * * \quad P$

goal setting ** P

Procedure for

individual written rehabilitation plan $\quad * * \quad S$

regular team meetings with patients $\quad * \quad$ S

written individual plan for follow-up * $\quad$ S

Patient received

a written rehabilitation plan

a written individual plan for follow-up

** $\quad \mathrm{P}$

Weekly evaluation of the rehabilitation plan P

Team meetings with possibility for the patient, next of kin and external personnel to participate

Length of rehabilitation stay

Improvement in $\mathrm{HRQOL}^{\mathrm{d}}$

Improvement in function

Goal attainment

(1)


Table 2 Main themes of quality and corresponding quality domains. Results of the first two voting rounds (Continued)

\begin{tabular}{|c|c|c|c|}
\hline Main themes of quality and corresponding references & Proposed quality domains & Ratinge & Cate-gory \\
\hline \multirow[t]{2}{*}{$31,34,37,38]$} & Satisfied with the rehabilitation & * & $\mathrm{O}$ \\
\hline & Health complications or negative events & * & $\mathrm{O}$ \\
\hline
\end{tabular}

${ }^{a}$ Structure ${ }^{b}$ Process ${ }^{c}$ Outcome ${ }^{d}$ Health Related Quality of Life ${ }^{e}$ the quality domains are marked according to the results of the ratings, round 1 and 2: ${ }^{* *}$ Appropriate * Uncertain. Not marked: Inappropriate

19 quality domains to be included for pilot testing, seven structure, seven process and five outcome domains (Table 2. Figure 1).

\section{Pilot testing}

The expert panel operationalized the quality domains into 19 structure, 12 process and five outcome QIs (Fig. 1).

A total of 29 rehabilitation units located in all regions of Norway agreed to participate in the pilot test; 15 from specialist care (10 rehabilitation institutions and five hospital departments) and 14 from primary care (five institutional, seven home based and two day units).

A total of 207 patients received the patient questionnaire, of whom 164 (79\%) completed it. Forty-one did not answer repeated phone calls, and two were excluded due to cognitive impairment. In five of the units, no patients answered the questionnaire.

The majority of participants were female and admitted to rehabilitation at a specialist rehabilitation institution. They had one or several of the following diagnoses: inflammatory rheumatic disease $(n=115)$, osteoarthritis $(n=75)$, neck-, shoulder- and/or back-pain $(n=86)$, generalized musculoskeletal pain $(N=44)$, fractures $(N=36)$, and osteoporosis $(n=24)$. More than half were employed and had previously undergone rehabilitation.
Compared to the participants in specialist care, participants in primary care were older and more predominantly lived alone. Other patient characteristics are shown in Table 3.

Mean time spent on answering the questionnaire was $6.0 \mathrm{~min}$ for managers and $5.5 \mathrm{~min}$ for patients, whereas mean (range) face validity scores were 8.3 (8) and 7.9 (9), respectively.

\section{Pass rates (PRs)}

Figure 2 shows the QIs and the corresponding PRs from the pilot test.

The PRs for structure QIs ranged from lowest 17\% for the QI Assessment of HRQoL 3-6 months after discharge to highest $96 \%$ for the QIs Defined patient target group and Assessment of adverse events.

The PRs for process QIs ranged from lowest 19 and $22 \%$ for the QIs Access to meetings for next of kin and external personnel and External personnel were involved in planning follow-up, to highest 93\% for the QI Initial health assessment and 91\% for the QI Participated in setting goals.

The PRs for the five outcome QIs ranged from 77 to $89 \%$.

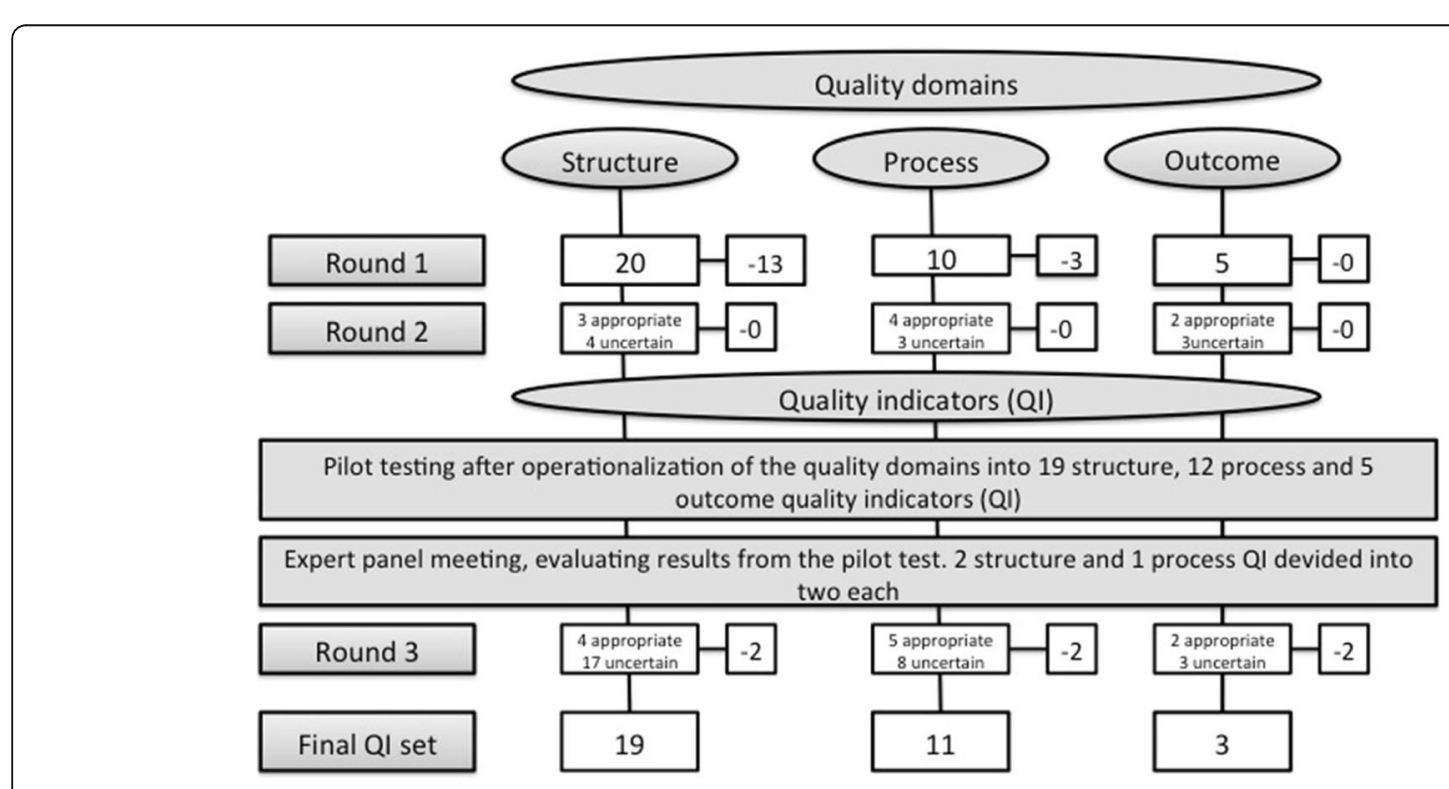

Fig. 1 The consensus process and ratings of the quality domains and indicators 
Table 3 Characteristics of 164 patients participating in pilot testing of the RMD rehabilitation QI set

\begin{tabular}{|c|c|c|c|c|c|}
\hline & \multirow[t]{3}{*}{ Total } & \multirow[t]{3}{*}{ Missing } & \multicolumn{3}{|c|}{ Rehabilitation in } \\
\hline & & & \multicolumn{2}{|l|}{ Specialist Care } & \multirow{2}{*}{$\begin{array}{l}\text { Primary Care } \\
\text { Munici- pality }\end{array}$} \\
\hline & & & Hospital & Rehab institution & \\
\hline N (\%) & $164 /(100)$ & $6(4)$ & $33(20)$ & $91(55)$ & $34(21)$ \\
\hline Female gender, N (\%) & $126(77)$ & 6 & $22(67)$ & $81(89)$ & $23(68)$ \\
\hline Age, mean(SD) & $60(16)$ & 6 & $54(14)$ & $55(12)$ & $77(13)$ \\
\hline Living alone, N (\%) & $59(36)$ & 6 & $10(30)$ & $31(34)$ & $18(53)$ \\
\hline > 12 years of education, $\mathrm{N}(\%)$ & $80(49)$ & 10 & $19(58)$ & $47(52)$ & $14(41)$ \\
\hline Employment, N (\%) & $87(53)$ & 0 & $23(70)$ & $64(70)$ & $0(0)$ \\
\hline Previous rehabilitation, N (\%) & $86(52)$ & 8 & $23(70)$ & $43(47)$ & $20(59)$ \\
\hline $\begin{array}{l}\text { One/more than one co-morbidity that could influence the rehabilitation } \\
\text { process, } \mathrm{N}(\%)\end{array}$ & $70(43) / 23(14)$ & $9 / 66$ & $16(48) / 5(15)$ & $38(42) / 15(16)$ & $16(47) / 3(9)$ \\
\hline
\end{tabular}

Figure 3 shows the variations in sum PRs for the QIs. Two of the rehabilitation units reached a $100 \%$ sum PR for the structure indicators, and four units reached $\leq 25 \%$. $4 \%$ of the patients reached a $100 \%$ sum PR of the process indicators, and $11 \%$ reached $\leq 25 \%$. A total of $70 \%$ of the patients passed all three outcome indicators Improvement in quality of life, Important improvement in physical, psychological or social function and Reached important goals. 13.8\% passed one or no outcome indicator.
Results for rehabilitation unit number 11, 19, 22, 26 and 27 (Fig. 3) are not given in the process and outcome diagrams, since no patients in these units answered the questionnaire.

The mean (range) sum PRs for the structure, process and outcome QIs were 59\%(84\%), 66\%(100\%) and $84 \%(100 \%)$, respectively.

In general, the mean sum PRs were higher in specialist compared to primary care; for structure QIs 64.2 and $58.4 \%(p=0.3)$, and for process QIs 70.0 and 50.0\%,

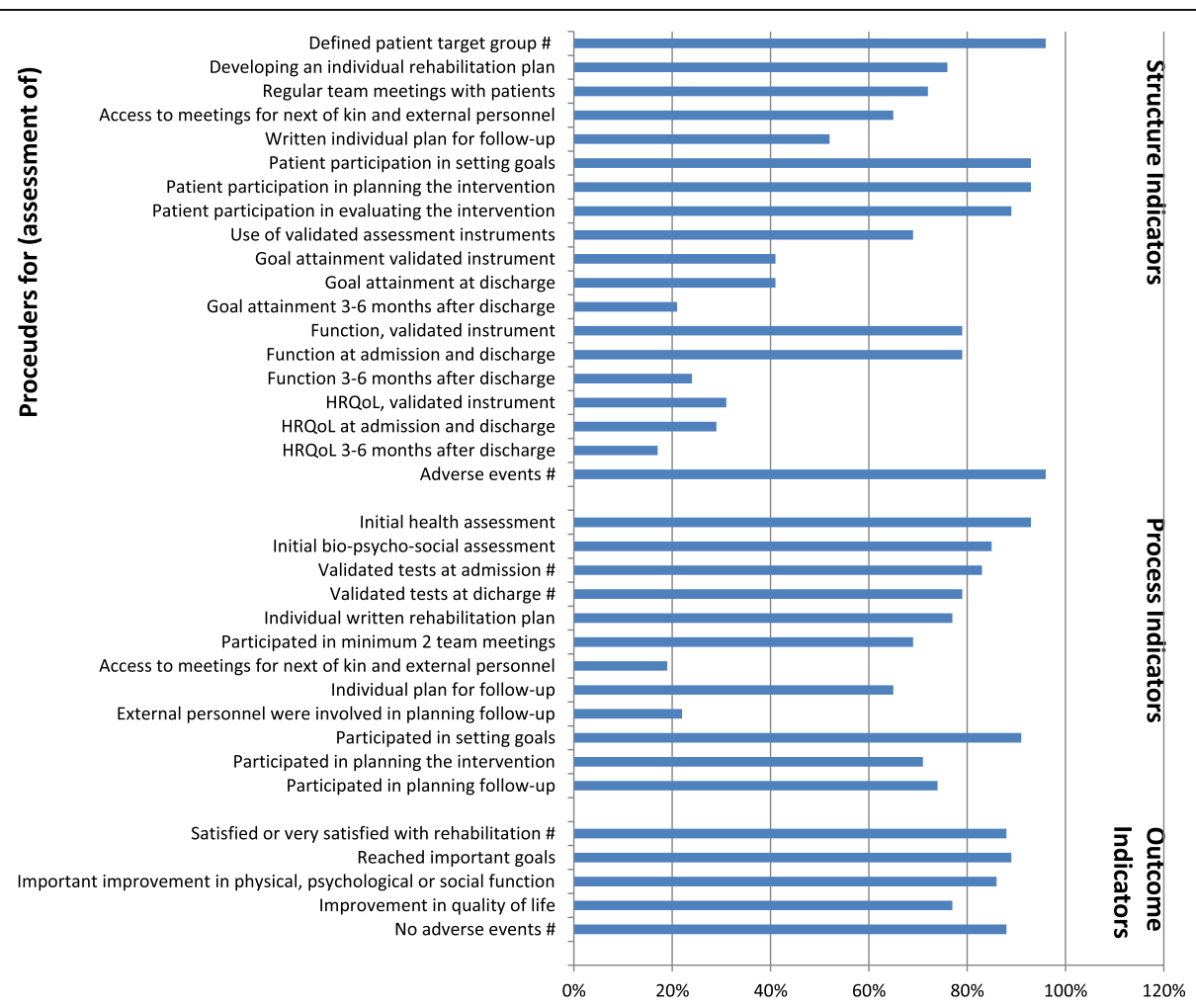

Fig. 2 The Qls and corresponding PRs. The Qls rejected in round 3 are marked with \# 

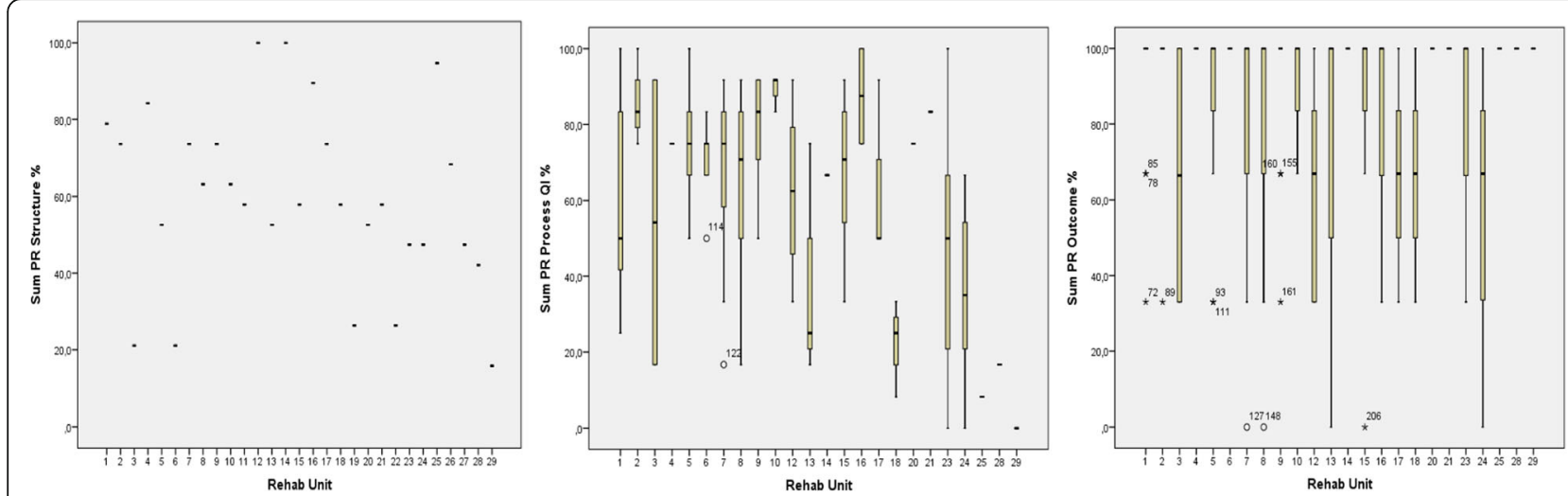

Fig. 3 Variations in PRs visualized in a point diagram for the structure Qls, and boxplots for the process and outcome Qls. Specialist rehabilitation units are unit number $1,2,5,6,7,8,9,10,11,12,13,16,17,18$, and 21 . The other units' numbers are primary care

$(p=0.001)$, respectively. Regarding outcome QIs the mean sum score was $83.3 \%$ in specialist care and $80.0 \%$ in primary care $(p=0.5)$.

The total mean sum PRs of the process and outcome QIs correlated strongly and positively to the PR of the outcome indicator Satisfied or very satisfied with the rehabilitation $(\mathrm{r}=0.56 p<0.001) .75 \%$ of the patients who passed the outcome QI Important improvement in function also passed the outcome QI Improvement in quality of life.

Regarding comments to the questionnaires, three patients and two managers commented on comprehensibility of some of the QIs, and four patients said it was difficult to answer only "yes" or "no".

\section{Reaching consensus on the final QI set}

Evaluation of the QIs after the pilot test resulted in dividing three of the QIs to ensure accurate answers to each question (Fig. 1). This concerned the structure and process indicators Access to meetings for next of kin and external personnel and the structure indicator Procedures for an individual plan for follow-up.

The third round (voter participation $72 \%$ ) resulted in exclusion of the structure indicators Defined patient target group and Assessment of adverse events, the process indicators Validated tests at admission and at discharge, and the outcome indicators Satisfied or very satisfied with the rehabilitation and No adverse events, all marked with \# in Fig. 2.

An additional file shows the final approved QI set (see Additional file 1).

\section{Discussion}

The primary aim of this project was to develop and pilot test a QI set for use in rehabilitation of people with RMDs. To ensure a transparent and scientific process, we adhered to the Rand/UCLA Appropriateness Method entailing that a broad expert group met several times and engaged in anonymous voting rounds.

The nineteen of the 35 proposed quality domains, which had been rated appropriate or uncertain in the voting rounds, were operationalized into 19 structure, 12 process and five outcome QIs formulated as yes/no questions. The pilot testing in 29 rehabilitation units and by 164 patients was followed up with an evaluation of the test results and a final voting round of the uncertain QIs in the expert panel, which resulted in 19 structure, 11 process and three outcome QIs.

When we updated the literature search, two new quality indicator sets and a systematic review of quality measures were detected [16-18]. Even though these sets to some degree align with the present, they are more pointed towards specific RMDs. Thus, to our knowledge, the present QI set is still the first one developed to measure quality in rehabilitation covering the whole RMD group and both structure, process and outcome quality and using appropriate methodology. Considering that RMDs have become the main contributor to disability in the Western world [1], assessing the quality of care will become increasingly important to policy-makers, providers, and patients.

The pilot revealed substantial potential for improvement in several important quality domains for many participating units. Three domains stood out. The first was Evaluation meetings along the rehabilitation process with possibility to participate for the patient, next of kin and external personnel. This domain was developed into both structure indicators (mean sum PR from 65 to $72 \%$ ), and process indicators (mean sum PR from 18 to $70 \%$ ). The domain represents a basic principle in rehabilitation [39]. Patient-participation and self-determination are crucial to the outcome and quality of rehabilitation [40]. However, our results support governmental reports documenting that patient-participation in rehabilitation is less than optimal [41]. The importance of family involvement is also 
well known [42]. Both the low PRs and the normative value of this domain indicate that it is an appropriate component of a QI set for rehabilitation.

The second domain was Use of validated assessment instruments at admission, discharge and 3-6 months after rehabilitation. This domain was developed into ten structure indicators (mean sum PRs from 17 to 69\%). Standardized assessment is the first step in, and a fundamental part of, a rehabilitation process $[8,43]$. At the individual level, it is essential as a basis for setting rehabilitation goals, planning interventions, and monitoring and evaluating effects over time. On a group level, assessment is important for evaluating and comparing results of various rehabilitation programmes, to develop and improve programmes and to evaluate cost-effectiveness [44]. Again the low scores in the pilot and the normative value of the domain indicate that this is an appropriate component of a QI set for rehabilitation.

The third domain with a notable potential for improvement was Individual planning of follow-up, involving the patient and external personnel. This domain was developed into both a structure indicator (mean sum PR 52\%), and process indicators (mean sum PRs from 25 to 65\%). The lack of coordination and cooperation between rehabilitation programmes and levels of care has been documented for years as one of the weakest elements in rehabilitation $[8,45]$, and there are unfortunately few signs of improvement. When discharged from rehabilitation, patients too often feel isolated and poorly prepared for living with disability in the municipality and know too little about where to turn to access the appropriate further health care [45]. These results underscore the importance of this quality domain and indicate that a shift is still needed in the field of rehabilitation, away from the acute-illness, curative model to one that acknowledges the long-term nature of a life with chronic disability.

Overall face validity of the QI set was tested with one question in the pilot study. However, during the anonymous ratings, panel members scored proposed items according to whether it was an obvious measure of quality, if it measured the intended item, and if it captured significant aspects of health and disease or important not medical conditions valued by the patients [23]. Thus, validation of each item was an inherent part of the consensus process. However, checking visits at participating centres, and controlling for effects of different patient characteristics is suggested for further validation of the QI set.

A limitation of the proposed QI set is that it is based on self-reports from rehabilitation unit leaders and users/patients and lacks data traditionally perceived as structural indicators. For practical application and information about the resource use of the health care, the proposed QIs should be linked to such data, i.e. national registers on work participation or medication, in future studies.

Regarding the feasibility of the QI set, the simple yes/no questions and the short time both managers and patients spent on answering them are in congruence with claims that data collection for quality measurement must be simple and not resource demanding [26]. However, while the participants in this pilot test answered the questionnaires by telephone, the intention is to manage the QIs as a self-reported questionnaire. This might influence both the answers given and the time to complete.

Recall bias may also have influenced the results, since the questions were answered one to two months after the rehabilitation period. On the other hand, these answers may be more reliable, as the first emotional reaction after the rehabilitation has come to some distance, and patients have got time to test their goals and self-management strategies in their home environment.

While the objective of the project was to develop a QI set for use in rehabilitation of patients with RMDs, the QI set appears generic and overriding and not limited to specific disease groups. This can be explained by the fact that good quality in rehabilitation encompasses change processes aimed at enhancing activity, participation and quality of life, which to a large extent are generic and independent of diagnostic group [46, 47]. The broad expertise in the consensus group and the fact that the systematic literature searches were not limited to RMDs may also have contributed to the perceived generic nature of the set. This may increase its feasibility, since rehabilitation takes place largely in units with mixed disease groups, especially in primary care.

Since the basic principles of rehabilitation to a large degree are the same across Europe and often independent of diagnostic group, it would be useful to try to reach international consensus for a generic QI set, which could be used to compare the quality of rehabilitation across countries. Further, diagnosis specific health care quality indicators, such as the eumusc.net sets for rheumatoid arthritis and osteoarthritis [13, 14], may be combined with the present set in studies focusing on specific diagnostic groups.

The healthcare level at which rehabilitation takes place varies between countries. A contemporary policy in many countries is to move responsibility for rehabilitation from specialist to primary care. To be able to monitor the quality of patient rehabilitation trajectories over time and compare the quality at different care levels, we aimed at developing a QI set that is valid and feasible both in a specialist and primary health care context. One potential risk of this strategy may be a ceiling effect 
in specialist care, since higher quality is expected at this level. Still, the results from the pilot test indicate considerable potentials for improvement at both levels.

The development of two separate questionnaires answered by rehabilitation managers and patients, respectively, may help to shed light on the black box of rehabilitation [48]. Analysing associations between structure, process and outcome indicators may identify factors of special importance to achieve positive outcomes. The matching of several of the structure and process indicators also allows for monitoring and comparing quality from both a system and user perspective, and for individual rehabilitation units as well as in a larger context. The pilot test showed clear differences in PRs for several of the matched indicators. However, the small scale testing does not justify for firm conclusions regarding these items.

A limitation in the pilot test was that the participating rehabilitation units were not randomly selected and were predominantly expected to give rehabilitation of high quality. This selection bias may have resulted in higher PRs than are representative for the average Norwegian rehabilitation unit. Furthermore, the present QI set was developed and pilot tested in a Norwegian context. As far as we know, the content of Norwegian rehabilitation of RMDs is largely comparable to other Northern European countries [30]. Nevertheless, little is known about the Norwegian level of quality in rehabilitation of RMDs compared to other countries. The pilot testing of the QI set was small scale with the mandatory uncertainties of such studies. The validity of the QI set should therefore be tested in other settings and larger samples. To explore the perceived generic nature of the QI set, it should also be examined in mixed diagnostic groups.

\section{Conclusion}

A QI set for the rehabilitation of RMDs has been developed and pilot tested according to the Rand/UCLA Appropriateness Method. The results from the pilot test indicate that the QI set has good overall face validity and is feasible for monitoring quality in rehabilitation. The large variation in PRs suggests a potential for quality improvement in rehabilitation.

\section{Additional file}

Additional file 1: The final approved QI set. (DOCX 25 kb)

\section{Abbreviations \\ PR: Pass rate; QI: Quality indicator; RMD: Rheumatic and Musculoskeletal Disease}

\section{Acknowledgements}

The authors would like to thank the consensus panel members outside the author group for their significant work in the developing process; Olav
Teigen, patient representative, The Norwegian Back Association, Guro Lilleas, municipality of Oslo, Camilla Michelsen, municipality of Larvik, Aase Skarbø, Hospital for Rheumatic Diseases, Lillehammer, Siri Skumlien, The Norwegian LHL Hospitals, Bente M Joten Skog, municipality of Ullensaker, Ellen Torgersen, municipality of Kristiansand.

Sincere thanks also to the librarian Nina Jorgensen for conducting the literature searches and to Aase Frich, who did pilot test telephone interviews. Finally, our gratefulness to all patients and rehabilitation managers who participated in the pilot testing.

\section{Funding}

The project was funded by the Norwegian Health Directorate. The Norwegian Health Directorate had no role in the design of the project, in collection, analysis, and interpretation of data or in writing the manuscript.

\section{Availability of data and materials}

The datasets generated and analyzed during the current study are not publicly available, since such availability was not included in the request for approval from the Data Inspectorate/Data Protection Official, but are available from the corresponding author on reasonable request.

\section{Authors' contributions}

IJ read the titles of the articles from the literature searches, the relevant abstracts and full text papers and drafted the reference list and the fact sheets for the expert panel. She prepared, collected and analysed the data from the expert voting rounds and the pilot test (a study secretary collected $40 \%$ of the data from the patients). She drafted the manuscript for publication in close collaboration with IK, MK and KBH. IJ discussed the expert panel reference list with IK and MK. IJ, IK and MK edited the fact sheets of the quality domains before they concluded on final drafts. IJ presented and discussed the results from the voting rounds and the pilot test with $\mathbb{I K}$ and MK before they were presented for the expert panel. AA, $A H, J B B, T G, K B H, U H, K A L, M L, J E N$, IO, SHW were members of the expert panel and contributed to the quality domain list, participated in the inperson meetings and voting rounds, operationalization of the quality domains into quality indicators. AK set up the electronic voting forms for each voting round. All authors read the drafts of the article and approved the manuscript.

\section{Ethics approval and consent to participate}

The original study which the current study originated from was presented for the Regional Ethic Committee in South-East in June 2015. Their decision was that the project fell outside the scope of the Health Research Act, cf. section 2, and therefore could be implemented without approval by the Regional Ethical Committee. The project was thus sent to the Data Protection Official at Oslo University Hospital, who approved the original project (ref.no: 2015/16099). In June 2016, an additional request was sent to the Data Protection Officer regarding development of the Quality Indicator Set and the additional data collection in the original project. The additional data collection was approved in August 11th 2016 (same ref.no as the previous decision).

All participants provided written consent to participate in the pilot test.

\section{Consent for publication}

Not applicable.

\section{Competing interests}

The authors AA, JBB, TG, UH, AH, KAL, ML, JEN, SHW, IO and KBH were a part of the study as members of the expert panel, which is a potential conflict of interest. The other authors declare that they have no competing interests.

\section{Publisher's Note}

Springer Nature remains neutral with regard to jurisdictional claims in published maps and institutional affiliations.

\section{Author details \\ ${ }^{1}$ National Advisory Unit on Rehabilitation in Rheumatology, Diakonhjemmet Hospital, P.O. Box 23, 0319 Oslo, Norway. ${ }^{2}$ Department of General Practice, Institute of Health and Society, Faculty of Medicine, University of Oslo, P.O. Box 1130 Blindern, 0318 Oslo, Norway. ${ }^{3}$ Department of Rehabilitation, University Hospital of North Norway, Tromsø, Norway. ${ }^{4}$ Faculty of Health Sciences, Department of Clinical Medicine, UiT The Arctic University of Norway, Tromsø, Norway. ${ }^{5}$ National Advisory Unit on Occupational}


Rehabilitation, Haddlandsvegen 20. 3864, Rauland, Norway. ${ }^{6}$ Uni Research Health, Nygårdsgaten 112, 5008 Bergen, Norway. ${ }^{7}$ Unicare Jeløy Rehabilitation Centre, Bråtengaten 94, N-1515 Moss, Norway. ${ }^{8}$ Norwegian Rheumatism Association, PO Box 2653, Solli, N-0203 Oslo, Norway. ${ }^{9}$ Sunnaas Rehabilitation Hospital, 1453 Bjørnemyr, Norway. ${ }^{10}$ Department of Physical Medicine and Rehabilitation, St. Olavs Hospital, Trondheim University Hospital, Trondheim, Norway. ${ }^{11}$ The Municipality of Sandefjord, PO Box 2025, 3202 Sandefjord, Norway. ${ }^{12}$ Rehabilitering Vest AS, PO Box 2175, 5504 Haugesund, Norway. ${ }^{13}$ Centre for Quality Improvement and Development, University Hospital of North Norway, Box 20, N-9038 Tromsø, Norway.

\section{Received: 19 October 2018 Accepted: 10 April 2019} Published online: 29 April 2019

\section{References}

1. Murray CJL, Vos T, Lozano R, Naghavi M, et al. Disability-adjusted life years (DALYs) for 291 diseases and injuries in 21 regions, 1990-2010: a systematic analysis for the global burden of disease study 2010. Lancet. 2012;380:2197-223.

2. Al Maini M, AdeloWo F, Al Saleh J, et al. The global challenges and opportunities in the practice of rheumatology: white paper by the world forum on rheumatic and musculoskeletal diseases. Clin Rheumatol. 2015;34: 819-29.

3. Hochberg MC, Altman RD, April KT, et al. American College of Rheumatology 2012 recommendations for the use of nonpharmacologic and pharmacologic therapies in osteoarthritis of the hand, hip, and knee. Arthritis Care Res (Hoboken). 2012;64:465-74.

4. Carville SF, Arendt-Nielsen L, Bliddal H, et al. EULAR evidence based recommendations for the management of fibromyalgia syndrome. Ann Rheum Dis. 2008;67:536-41.

5. Kamper SJ, Apeldoorn AT, Chiarotto A, Smeets RJ, Ostelo RW, Guzman J, et al. Multidisciplinary biopsychosocial rehabilitation for chronic low back pain. Cochrane Database Syst Rev. 2014;2(9):CD000963.

6. Kjeken I, Bo I, Ronningen A, et al. A three-week multidisciplinary in-patient rehabilitation programme had positive long-term effects in patients with ankylosing spondylitis: randomized controlled trial. J Rehabil Med. 2013;45: 260-7.

7. Klokkerud M, Hagen K, Lochting I, Uhlig T, Kjeken I, Grotle M. Does the content really matter? A study comparing structure, process, and outcome of team rehabilitation for patients with inflammatory arthritis in two different clinical settings. Scand J Rheumatol. 2012;4:20-8.

8. Document 3:11 (2011-2012). The Office of the Auditor General's investigation into rehabilitation in the health service; 2012. ISBN 978-828229-163-7.

9. The Health\&Care21 Strategy. Research and innovation in health and care (2015-2018). The Research Council of Norway; 2014. ISBN 978-82-99002.

10. Mainz J. Developing evidence-based clinical indicators: a state of the art methods primer. Int J Qual Health Care. 2003;15(Suppl 1):i5-i11.

11. Lawrence M, Olesen F. Indicators of quality in health care. Eur J Gen Pract. 1997;3(3):103-8.

12. Mainz J. Defining and classifying clinical indicators for quality improvement. Int J Qual Health Care. 2003;15:523-30.

13. EUMUSC.net. Health care quality indicators for OA. 2013. http://www. eumusc.net/myUploadData/files/

EUMUSC\%20Health\%20care\%20quality\%20indicators\%20for\%200A\%20KE. pdf access date 12 Mar 2019.

14. EUMUSC.net. Health care quality indicators for RA. 2013. http://www. eumusc.net/myUploadData/files/

EUMUSC\%20Health\%20care\%20quality\%20indicators\%20for\%20RA\%20KE.pdf access date 12 Mar 2019

15. Strombeck B, et al. Health care quality indicators on the management of rheumatoid arthritis and osteoarthritis: a literature review. Rheumatology (Oxford). 2013;52(2):382-90.

16. Peter WF, Hurkmans EJ, van der Wees PJ, Hendriks EJM, van Bodegom-Vos $L$, Vliet Vlieland TPM. Healthcare quality indicators for physiotherapy Management in hip and Knee Osteoarthritis and rheumatoid arthritis: a Delphi study. Musculoskelet Care. 2016;14:219-32.

17. Gratacós J, et al. Standards of care and quality indicators for multidisciplinary care models for psoriatic arthritis in Spain. Rheumatol Int. 2018;38:1115-24.
18. Cooper M, Rouhi A, Barber CEH. A systematic review of quality measures for inflammatory arthritis. J Rheumatol. 2018;45(2):274-83.

19. CARF International 2017. http://www.carf.org/

20. Melão N, Amorim M, Marimon F, Alegre I. EQUASS assurance certification: the view of Pioneer adopters. In: Proceedings of 2 nd international conference on quality engineering and management. Guimarães: ICQEM; 2016. p. 615-32. ISBN 978-989-20-6814-5.

21. Department of Health and Human Services OMB No. 0938-0842 Center for Medicare \& Medicaid Services. IRF-PAI (version 1.3). 2015. https:// federalregister.gov/d/2018-16517.

22. Skempes D, Melvin J, von Groote P, Stucki G, Bickenbach J. Using concept mapping to develop a human rights based indicator framework to assess country efforts to strengthen rehabilitation provision and policy: the rehabilitation system diagnosis and dialogue framework (RESYST). J Global Health. 2018;14(1):96.

23. Fitch K, Bernstein S, Aguilar MS, Burnand B, LaCalle JR, Lazaro P, et al. (RAND monograph report). The Rand/UCLA Apporpriateness method User's manual. Santa Monica: RAND; 2001. p. 1-123. ISBN 0-8330-2918-5

24. Boulkedid R, Abdoul H, Loustau M, Sibony O, Alberti C. Using and reporting the Delphi method for selecting healthcare quality indicators: a systematic review. PLoS One. 2011;6(6):e20476. https://doi.org/10.1371/journal.pone. 0020476.

25. Report No. 21 to the Storting. Responsibility and empowerment: towards a holistic rehabilitation policy. Oslo: Ministry of Health; 1999. St.meld. nr. 21 (1998-99) - regjeringen.no.

26. Kelley E, Hurst J. "Health care quality indicators project: conceptual framework paper". OECD health working papers, no. 23. Paris: OECD Publishing; 2006. https://doi.org/10.1787/440134737301.

27. Mokkink LB, Terwee CB, Patrick DL, Alonso J, Stratford PW, Knol DL, et al. The COSMIN study reached international consensus on taxonomy, terminology, and definitions of measurement properties for health-related patient-reported outcomes. J Clin Epidemiol. 2010 Jul;63(7):737-45. https:// doi.org/10.1016/j.jclinepi.2010.02.006.

28. The Norwegian Neck and Back Register. https://www.kvalitetsregistre.no/ registers/norsk-nakke-og-ryggregister.

29. Farin $\mathrm{E}$, et al. Quality assessment in rehabilitation centres: the indicator system 'Quality profile. Disabil Rehabil. 2004;26(18):1096-104.

30. Grotle $\mathrm{M}$, et al. What's in the black box of arthritis rehabilitation? A comparison of rehabilitation practice for patients with inflammatory arthritis in northern Europe. J Rehabil Med. 2013;45(5):458-66.

31. Klokkerud $\mathrm{M}$, et al. Development of a framework identifying domains and elements of importance for arthritis rehabilitation. J Rehabil Med. 2012;44(5): 406-13.

32. Van der Weide WE, Verbeek JHAM, van Dijk FJH. Relation between indicators for quality of occupational rehabilitation of employees with low back pain. Occup Environ Med. 1999;56:488-93.

33. AAPMR, Standards for assessing medical appropriateness criteria for admitting patients to rehabilitation hospitals or units AAPMR. www.aapmr. org. https://docplayer.net/543995-Standards-for-assessing-medicalappropriateness-criteria-for-admitting-patients-to-rehabilitation-hospitals-orunits.html accessed 12 Mar 2019.

34. Edwards JJ, Khanna M, Jordan KP, Jordan JL, Bedson J, Dziedzic KS. Quality indicators for the primary care of osteoarthritis: a systematic review. Ann Rheum Dis. https://doi.org/10.1136/annrheumdis-2013-203913.

35. Li CL, Sayre EC, Kopec JA, Esdaile JM, Bar S, Cibere J. Quality of nonpharmacological Care in the Community for people with knee and hip osteoarthritis. J Rheumatol. 2011;38(10):2230-7. https://doi.org/10.3899/ jrheum.110264.

36. Grossman JM, MacLean CH. Quality indicators for the Management of Osteoporosis in vulnerable elders. Ann Intern Med. 2001;135:722-30.

37. Dy SM, Pfoh ER, Salive ME, Boyd CM. Health-related quality of life and functional status quality indicators for older persons with multiple chronic conditions. J Am Geriatr Soc. 2013;61(12):2120-7. https://doi.org/10.1111/jgs.12555.

38. Grande G, Romppel M. Subjective concepts of patients, physicians and social workers about quality in cardiac and orthopaedic rehabilitation. Psychother Psychosom Med Psychol. 2008;58(2):51-7.

39. Meyer T, Gutenbrunner C, Bickenbach J, Cieza A, Melvin J, Stucki G. Towards a conceptual description of rehabilitation as a health strategy. J Rehabil Med. 2011;43:765-9. 
40. Peoples H, Satink T, Steultjens E. Stroke survivors experiences of rehabilitation: a systematic review of qualitative studies. Scand J Occup Ther. 2011;18(3):163-71.

41. Ministry of Health and Care Services (HOD). Intensifying plan for habilitation and rehabilitation (2017-2019). Offprint to Prop. 15 (2016217). https://www.regjeringen.no/contentassets/.../no/... opptrappingsplanrehabilitering.pdf

42. Loupis YM, Faux SG. Family conferences in stroke rehabilitation: a literature review. J Stroke Cerebrovasc Dis. 2013;22(6):883-93.

43. Wade DI. Evidence relating to assessment in rehabilitation. Clin Rehabil. 1998;12:183-6

44. Stucki G, Cieza A, Ewert T, Kostanjsek N, Chatterji S, ÜstÜn TB. Application of the international classification of functioning, disability and health (ICF) in clinical practice. Disabil Rehabil. 2002;24(5):281-2.

45. Cott C. Client-centred rehabilitation: client perspectives. Disabil Rehabil. 2004;26(24):1411-22.

46. Wade DI. Rehabilitation - a new approach. Part two: the underlying theories. Clin Rehabil. 2015;29(12):1145-54.

47. Wade DI. Rehabilitation - a new approach. Part four: a new paradigm, and its implications. Clin Rehabil. 2016;30(2):109-18.

48. Wade D. Research into the black box of rehabilitation: the risks of a type III error. Clin Rehabil. 2001:15:1-4.

Ready to submit your research? Choose BMC and benefit from:

- fast, convenient online submission

- thorough peer review by experienced researchers in your field

- rapid publication on acceptance

- support for research data, including large and complex data types

- gold Open Access which fosters wider collaboration and increased citations

- maximum visibility for your research: over $100 \mathrm{M}$ website views per year

At $\mathrm{BMC}$, research is always in progress.

Learn more biomedcentral.com/submissions 